\title{
Intervenções de terapeutas ocupacionais junto a idosos com doença de Parkinson*
}

\author{
The interventions used by occupational therapist \\ at the treatment of elderly with Parkinson's disease
}

\author{
Maria Helena Morgani de Almeida ${ }^{1}$, Gislaine Alves Cruz ${ }^{2}$
}

\begin{abstract}
ALMEIDA, M. H. M.; CRUZ, G. A. Intervenções de terapeutas ocupacionais junto a idosos com doença de Parkinson. Rev. Ter. Ocup. Univ. São Paulo, v. 20, n. 1, p. 29-35, jan./abr. 2009.

RESUMO: O terapeuta ocupacional junto ao idoso com DP objetiva prevenir e reduzir perdas funcionais e, quadros de dependência e se utiliza basicamente de dispositivos tecnológicos, mudanças ambientais e técnicas adaptadas. Com o objetivo de conhecer intervenções de terapeutas ocupacionais com idosos com DP desenvolveu-se, em 2006, estudo qualitativo, exploratório e descritivo. Esse compreendeu aplicação de um roteiro de questões abertas, por meio de entrevistas a terapeutas ocupacionais com atuação na área de gerontologia; residentes e atuantes no Município de São Paulo. Sete terapeutas ocupacionais participaram do estudo. Referiram que os idosos enfrentam principalmente dificuldades motoras, cognitivas e emocionais e, orientam-se em suas intervenções por objetivos dos pacientes. Estes objetivos compreendem minimizar os sintomas, promover treino motor e destreza, resgatar e potencializar capacidades remanescentes, melhorar o desempenho nas atividades cotidianas, a independência e a qualidade de vida. As ações desenvolvidas incluem exercícios de coordenação motora, técnicas de relaxamento e respiração, atividades compensatórias e desenvolvimento de ações voltadas para as capacidades preservadas. Para o enfrentamento de distúrbios cognitivos, os entrevistados proporcionam desde estimulação cognitiva até simplificação de atividades. A atuação junto aos cuidadores e familiares é considerada fundamental para minimizar impactos decorrentes da progressão da doença. As abordagens utilizadas pelos entrevistados convergem para a preservação da independência, autonomia do idoso e uma vida com significado.
\end{abstract}

DESCRITORES: Idoso. Terapia ocupacional. Doença de Parkinson.

\footnotetext{
* Monografia apresentada para conclusão de Curso de Graduação em Terapia Ocupacional/ FM/USP.

1. Profa. Dra. do Curso de Terapia Ocupacional do Departamento de Fisioterapia, Fonoaudiologia e Terapia Ocupacional da FMUSP.

2. Terapeuta Ocupacional.

Endereço para correspondência: Maria Helena Morgani de Almeida, Departamento de Fisioterapia, Fonoaudiologia e Terapia Ocupacional da FMUSP. Rua Cipotânea, 51.05360-000. São Paulo, SP. E-mail: hmorgani@usp.br
} 


\section{INTRODUÇÃO}

$\mathrm{C}$ onsiderando-se as repercussões funcionais e psicossociais da doença de Parkinson (DP) especialmente sobre a pessoa idosa e as possibilidades de atuação do terapeuta ocupacional junto a esta população, desenvolveu-se em 2006, estudo com propósito de conhecer intervenções adotadas por alguns terapeutas ocupacionais, residentes e atuantes no Município de São Paulo, junto a idosos com doença de Parkinson (DP). Propõe-se, através do presente artigo, apresentar e discutir as distintas formas de intervenção referidas por esses profissionais.

O envelhecimento populacional é considerado um fenômeno mundial e, segundo Litvak (1990), esse processo aumenta a suscetibilidade a enfermidades crônicas e incapacidades. Dentre os males crônicos potencialmente incapacitantes, especialmente para idosos, está a DP. No Brasil essa doença configura-se como uma das principais causas neurológicas de incapacidade física em pessoas acima de 60 anos (CABRAL; STOPPE,1993).

A DP parece associar-se com o processo degenerativo de certas estruturas do sistema nervoso central e também com fator genético. Segundo Cabral e Stoppe (1993), a DP é uma doença neurodegenerativa ligada aos gânglios da base.

A partir do reconhecimento de que não há cura para a DP, os tratamentos administrados visam preservar neurônios remanescentes, atenuar os sintomas e melhorar a qualidade de vida da pessoa acometida.

Partindo-se do pressuposto de que a prevenção ou redução de perdas funcionais constitui-se como objetivo principal do terapeuta ocupacional em gerontologia, admite-se a essencialidade da atuação deste profissional no processo de reabilitação de idosos com DP, minimizando o grande risco de tornarem-se dependentes em sua vida cotidiana.

\section{Terapia Ocupacional com idosos portadores de Doença de Parkinson}

Segundo Mora (2003) a Terapia Ocupacional abarca o tratamento de pessoas que apresentam déficit em tarefas básicas do autocuidado como alimentação, higiene, deambulação e atividades instrumentais. A autora destaca que, ao aumentar a participação das pessoas idosas em atividades que lhes interessa, esse profissional tem um papel relevante em sua recuperação funcional global.

Tendo em vista a promoção ou desenvolvimento da autonomia e da independência, o terapeuta ocupacional pode utilizar-se de dispositivos tecnológicos, sugerir mudanças ambientais e facilitar a execução de tarefas diversas tornando-as mais efetivas, seguras, econômicas, confortáveis e prazerosas. Esse profissional pode treinar a pessoa em habilidades, tais como força, percepção visual, coordenação, habilidades cognitivas, dentre outras bem como, nas atividades ocupacionais, para as quais apresenta dificuldades, integrando habilidades intactas e deficitárias (ALMEIDA, 2003).

Aragon (2008) indica técnicas para facilitar a realização de atividades, e que incluem método alternativo para sua realização, mudanças ambientais como reorganização de móveis, prescrição e orientação para aquisição de equipamentos de auto-ajuda e, aconselhamento para acessar serviços ou outra forma de suporte social.

Propondo métodos que simplifiquem a realização de tarefas diárias, a Terapia Ocupacional ajudará as pessoas com DP a melhorar a efetividade na realização destas tarefas, ajudando-as a superar os principais efeitos dos sintomas da doença no dia a dia. Ao proporcionar a pessoa com DP, o envolvimento e a realização de atividades significativas, o terapeuta ocupacional contribuirá para a promoção da sensação de autocontrole, autoeficácia e satisfação, intervindo desta forma para a redução de alguns efeitos de doenças que geralmente estão associadas à DP, como demência e depressão (MORA, 2003; ALMEIDA, 2005).

\section{OBJETIVO DO ESTUDO, MATERIAL E MÉTODO}

Com o propósito de conhecer intervenções adotadas por terapeutas ocupacionais junto a idosos com DP e orientando-se por princípios éticos que norteiam pesquisas com seres humanos (Resolução 196/96, CNS), desenvolveu-se em 2006, estudo qualitativo, exploratório e descritivo com uma amostra de conveniência composta por terapeutas ocupacionais. A partir da aplicação de um roteiro de questões abertas, por meio de entrevistas individuais, buscou-se levantar aspectos relevantes da atuação do terapeuta ocupacional junto a idosos com DP. Para a análise das entrevistas, optou-se pela análise temática de conteúdo (BARDIN, 1977).

Adotaram-se os seguintes critérios para a participação dos profissionais no estudo: a) formação em terapia ocupacional; b) com atuação, durante o estudo, na área de gerontologia; c) residentes e atuantes no Município de São Paulo; d) com interesse expresso em participar do estudo. 


\section{RESULTADOS E DISCUSSÃO}

Sete terapeutas ocupacionais foram contatados e con- cordaram em participar da pesquisa. O Quadro 1 apresenta um breve perfil dos entrevistados e das instituições em que prestam atendimento aos idosos.

QUADRO1. Breve perfil dos entrevistados e das instituições em que prestam atendimento aos idosos, São Paulo, 2006

\begin{tabular}{|c|c|c|}
\hline Variáveis & Categorias & Freq. \\
\hline Tempo de formação & $\begin{array}{l}4 \text { anos } \\
\text { entre } 10 \text { e } 15 \text { anos } \\
\text { mais de } 15 \text { anos }\end{array}$ & $\begin{array}{l}4 \\
2 \\
1\end{array}$ \\
\hline Experiência profissional & $\begin{array}{l}\text { Atuam com idosos incluindo aqueles acometidos por DP } \\
\text { Atuam especificamente com idosos acometidos por DP }\end{array}$ & $\begin{array}{l}5 \\
2\end{array}$ \\
\hline $\begin{array}{l}\text { População atendida pela Insti- } \\
\text { tuição }\end{array}$ & $\begin{array}{l}\text { Idosos } \\
\text { Pessoas com DP } \\
\text { Pessoas em processo de reabilitação }\end{array}$ & $\begin{array}{l}5 \\
1 \\
1\end{array}$ \\
\hline $\begin{array}{l}\text { Fontes de encaminhamento para } \\
\text { instituição }\end{array}$ & $\begin{array}{l}\text { Profissionais da saúde } \\
\text { Instituições } \\
\text { Familiares } \\
\text { Usuários }\end{array}$ & $\begin{array}{l}6 \\
3 \\
2 \\
2\end{array}$ \\
\hline
\end{tabular}

Segundo os entrevistados, os problemas enfrentados pelos idosos acometidos pela DP abrangem diversas esferas: funcional, ocupacional, relativos à saúde física e mental, social e econômica.

No que se referem à funcionalidade, os idosos com DP enfrentam dificuldades motoras e cognitivas, relacionadas às atividades básicas e instrumentais de vida diária e ao lazer. Conforme Nitrini (2003) a DP compreende quadro clínico constituído, basicamente, por tremor, acinesia, rigidez e instabilidade postural, mas pode também incluir outras alterações motoras como a acinesia súbita ou congelamento (freezing) e cinesia paradoxal (melhora súbita e de curta duração do desempenho motor), distúrbios mentais, demência, depressão e, autonômicos como obstipação intestinal, seborréia e tendência à hipotensão. Em conjunto com os sintomas motores, estes distúrbios são capazes de interferir, significativamente, na rotina da pessoa com DP transformando sua relação com o ambiente.

Os sintomas depressivos ocorrem em 40 a $50 \%$ das pessoas com DP, podendo ser reativos à doença ou compor sua sintomatologia (STOPPE, 1997). Sua intensidade pode variar de quadros leves a graves, em que a depressão é o sintoma mais importante e um dos fatores determinantes de incapacidade.

Alterações emocionais como insegurança e atitudes negativistas diante de situações novas também são comuns. As pessoas podem reagir a estas situações evitando sair de casa, ter contatos sociais e experimentar a adoção de novos hábitos. Alguns perdem a motivação e se tornam excessivamente dependentes dos familiares. Segundo um dos entrevistados:

"Uma das dificuldades é o fato de que não se dão conta de que seu corpo pode reagir beneficamente a partir de mudanças de atitudes e da introdução de hábitos diferentes e simples em suas rotinas."

Em sua intervenção, os terapeutas ocupacionais orientam-se prioritariamente por objetivos, desejos e necessidades expressos pelos idosos, embora afirmem que os idosos tenham muita dificuldade em formular estes objetivos, requerendo constante incentivo e estimulação para isto. Os profissionais orientam-se ainda, em suas intervenções, por referenciais teóricos e metodológicos.

De acordo com Aragon (2008) o terapeuta ocupacional deverá orientar seu plano de ação por atividades que a pessoa precise e queira realizar, ou seja, deverá traçar este plano em colaboração com a pessoa. Ainda segundo a autora, esse profissional estará almejando avaliar, identificar e intervir sobre as principais prioridades do indivíduo.

De maneira geral, os objetivos de intervenção, apresentados pelos entrevistados compreendem minimizar os sintomas decorrentes da doença, promover treino motor e destreza, resgatar e potencializar capacidades remanescentes, melhorar o desempenho nas atividades cotidianas, a independência e a qualidade de vida.

Cinco terapeutas procuram minimizar os sintomas da doença tendo como base as dificuldades apresentadas pelos 
idosos e dois terapeutas dirigem-se para o desenvolvimento de capacidades remanescentes, conforme segue:

“...Eu olho pra aquela senhora e vejo assim quais são as capacidades remanescentes que ela tem e é ai que eu vou trabalhar,... “

Considera-se que a prática de atividades cotidianas significativas, orientadas pelo terapeuta ocupacional possa favorecer tanto a minimização dos sintomas quanto o uso de capacidades remanescentes e que, portanto, estes objetivos não sejam mutuamente excludentes. Neistadt e Seymour (1995) referem que a prática de atividades ocupacionais envolve atividades, tarefas e papéis dirigidos às metas funcionais da pessoa relacionando-se estreitamente às necessidades de sua vida cotidiana, além de oferecem a possibilidade de integrar habilidades intactas e deficitárias.

Um dos entrevistados ilustra a possibilidade de integrar estes objetivos em sua intervenção:

"Meu objetivo é tentar minimizar ao máximo possivel os sintomas da doença, através de diversas formas de tratamento, exercícios ou atividades compensatórias, adequação ambiental para tentar maximizar a independência do idoso"

Segundo a PDS (2008) o terapeuta ocupacional combina estes objetivos ao estabelecer como propósitos melhorar a função física do paciente e sua independência.

As intervenções adotadas pelos entrevistados se baseiam em dados de avaliação, obtidos por meio da aplicação de protocolos validados e/ou de roteiros concebidos pela instituição ou pelo terapeuta para orientar seu raciocínio clinico.

Três terapeutas ocupacionais adotam avaliações de caráter multidimensional produzidas nas instituições onde trabalham e um profissional adota avaliação especifica produzida pelo setor de terapia ocupacional, por considerálas suficientes para traçar seu plano de atenção. No último caso, o profissional afirma que:

\section{"poderia buscar outras coisas também".}

Três terapeutas, embora utilizem instrumentos padronizados, afirmam que estes não são suficientes para amparar sua atuação clínica. Ainda que reconheçam os benefícios do uso de instrumentos padronizados na produção de estudos, no compartilhamento de resultados e no estabelecimento de diretrizes de atuação, consideram que seu uso exclusivo limita a visão do terapeuta. Estes profissionais consideram que abordagens padronizadas e não-padronizadas não se opõem, sendo desejável que se integrem para maior aproximação à complexidade de cada caso.

"avaliações padronizadas e raciocínio clínico tem de andar juntos, um nunca pode substituir o outro."

“...Os protocolos não determinam o atendimento o que determina é o dia-a-dia com o paciente..."

Os profissionais que adotam instrumentos padronizados utilizam: Índice de Barthel, (MAHONEY; BARTHEL, 1965) Lawton (LAWTON; BRODY, 1969) Blessed (BLESSED et al., 1968) Geriatric Depression Scale - GDS (SHEIKH; YESAVAGE, 1986). Medida de Independência Funcional - MIF (GRANGER; HAMILTON, 1987), Disability Assessment for Dementia - DAD (GÉLINAS et al., 1999) e Older Americans Resources and Services OARS (FILLENBAUM, 1984).

Conforme Borsoi (2000) a adoção de instrumentos para a avaliação periódica de aspectos da memória, atenção, orientação - temporal e espacial - julgamento e resolução das atividades básicas e instrumentais de vida diária, amparam a definição e ajustes às atividades, em conformidade à evolução da doença.

Quatro dos sete terapeutas referem adotar a abordagem grupal em suas intervenções, sendo que dois destes mencionam também atenção individual. Os benefícios de um grupo de terapia ocupacional para melhora da independência funcional, redução dos sintomas físicos e motores e melhora da qualidade de vida de pessoas com DP foram relatados por Gauthier et al. (1987). Com base em comparação de dados de avaliação obtidos antes e após um programa de reabilitação em grupo, observou-se que os sujeitos mantiveram seu status funcional depois de 1 ano de participação no programa, demonstraram uma diminuição significante da bradicinesia e perceberam uma melhora significante em seu bem estar psicológico.

As ações desenvolvidas pelos terapeutas ocupacionais entrevistados compreendem exercícios de coordenação motora e função manual, técnicas de relaxamento e respiração, estimulação percepto-cognitiva, atividades compensatórias, adaptações ambientais e desenvolvimento de ações voltadas para as capacidades preservadas.

Depreende-se que os terapeutas adotam tanto técnicas voltadas ao treino de habilidades em déficits quanto voltadas às atividades ocupacionais. Estas técnicas, em conjunto, ajustam-se às abordagens denominadas: "corretiva" e "adaptativa". Segundo Neistadt e Seymour (1995), a primeira abordagem compreende o treino da pessoa nas habilidades em déficits e que componham as atividades ocupacionais, tais como força, percepção visual, coordenação, habilidades cognitivas, entre outras. A segunda abordagem compreende o treino da pessoa nas atividades ocupacionais, para as quais a pessoa apresenta dificuldades, integrando habilidades intactas e deficitárias. Gray (1998) refere que quando as metas ocupacionais são estabelecidas 
em conjunto com a pessoa há possibilidade de integrar as duas formas de abordagens, uma vez que o envolvimento do paciente em atividades ou ações significativas aumenta a oportunidade para transferências de habilidades.

Quatro entrevistados atendem casos iniciais de Parkinson com discretas perdas funcionais enquanto três possuem experiência no atendimento a idosos com perdas funcionais significativas. Em estágios iniciais da DP, os terapeutas apontaram ações voltadas para exercício de capacidades, manutenção da capacidade funcional e prevenção de perdas funcionais, além de ações voltadas para diminuir a insegurança trazida pela presença de uma doença degenerativa. Dentre os recursos adotados, as terapeutas relataram a prática de exercícios com vistas a manter o equilíbrio, amplitude de movimento e coordenação motora. Por meio de tais técnicas podem, além de preservar funções físicas e impedir um rápido declínio funcional, estimular funções sociais contribuindo para evitar a exclusão social da pessoa. Uma das entrevistadas afirmou utilizar técnicas de relaxamento e respiração para controle do tremor e da rigidez, considerando que esta prática auxilia o idoso a não "negligenciar" o próprio corpo, a reconhecer os sintomas da doença e exercer certo domínio sobre estas manifestações.

De acordo com Zaslavsky (2002) a verdadeira meta da prevenção deveria ser manter ótima a função adiando assim a inaptidão e promovendo apoio ao paciente e sua família. Chen e Neves (2002) sugerem ainda que a prevenção, dirigida ao idoso com Parkinson, seja direcionada à manutenção de cuidados básicos de saúde (conservação da higiene, alimentação, atividades e convivência social), orientação aos cuidadores e familiares e intervenções de reabilitação para minimizar os efeitos dos sintomas da doença.

Em estágios mais avançados da doença, junto a idosos que vivenciam rigidez e têm dificuldade, por exemplo, de virar-se no leito, os entrevistados relataram o uso de colchões de ar para aumentar o conforto noturno e adaptações no leito para a troca de decúbito, além de formas facilitadas para realizar esta e outras atividades, órteses nas mãos também são indicadas pelos terapeutas para evitar deformidades. De acordo com a PDS (2008), objetivando melhorar a função física do paciente e sua independência, o terapeuta ocupacional integra várias técnicas como dispositivos tecnológicos e formas facilitadas de realização de atividades.

Embora a maioria das pessoas com DP não apresente declínio intelectual, podem ocorrer distúrbios cognitivos como dificuldades de concentração e de memória para fatos recentes, dificuldades para cálculos e em atividades que requerem orientação espacial. Tais alterações costumam se intensificar com o avanço da doença, especialmente em pessoas idosas. Ainda que esses distúrbios não sejam suficientes para gerar dependência, costumam causar desconforto e preocupação para a pessoa e seus familiares, o que torna imperativo a estimulação cognitiva e o oferecimento de oficinas para treinamento de memória e funções relacionadas. Ressalta-se adoção destas práticas pelos entrevistados.

A depressão associada à doença crônica incapacitante é considerada pelos entrevistados uma enfermidade freqüente nos consultórios e instituições diversas que atendem idosos com Parkinson. Tal conhecimento, entretanto, não torna a presença da depressão um evento menos preocupante, devendo, desta forma, receber atenção do terapeuta quanto à sua evolução, à eficácia da medicação utilizada e na interrelação com outros profissionais de saúde. Um dos entrevistados salientou a necessidade de um bom diagnóstico, boa comunicação com o médico responsável e com outros profissionais vinculados ao caso, a fim de trocar informações sobre o idoso e manter a depressão controlada. Um dos terapeutas utiliza técnicas de relaxamento e atividades expressivas para intervenções em casos de depressão. Segundo a PDS (2008) atividades como arte, música e escrita são oportunidades para comunicar e compartilhar idéias e sentimentos, melhorar o humor, reduzir níveis de estresse bem como os sintomas motores da DP.

Nos casos de demência associada, que se sucedem em cerca de $20 \%$ das pessoas acometidas - (http://www. parkinson.med.br, 2005 nov. 8) -, os terapeutas apontaram a necessidade de simplificar atividades, estimular as funções cognitivas e estabelecer estreita relação com cuidadores e familiares para o sucesso da intervenção.

A atuação junto aos cuidadores e familiares é considerada fundamental por todos os participantes, contudo um deles não trabalha diretamente com esta população, visto que na instituição em que atua, esse trabalho é realizado por profissionais de outra área.

Os entrevistados afirmam depender do suporte de familiares e cuidadores para o êxito do tratamento. Para tanto, eles ou outros profissionais prestam orientações a estes cuidadores quanto à postura e procedimentos a serem adotados diante das mudanças comportamentais e físicas que o idoso vivencia a fim de minimizar os impactos decorrentes da progressão da doença. A prática descrita pelos entrevistados ampara-se pelo exposto pela PDS (2005), segundo a qual o sucesso no gerenciamento da DP depende da parceria com o indivíduo, seus cuidadores e a rede de profissionais envolvidos na atenção, sendo esta parceria favorecida pela promoção de informação e entendimento acerca da doença. 
Quanto às ações desenvolvidas pelo conjunto dos terapeutas ocupacionais entrevistados pode-se afirmar que são amparadas tanto por informações que dizem respeito à singularidade da pessoa com DP - como aquelas relativas à sua história de vida e ocupacional - quanto por aquelas referentes aos sintomas da DP e sua repercussão sobre a vida cotidiana. Em conjunto, estas informações amparam ações que auxiliam o idoso e a família a conhecerem e elaborarem as alterações por eles vivenciadas e, ainda, a construírem estratégias para melhorar o desempenho em atividades.

“...acredito que uma boa formação em TO (conceitos, objetivos, técnica e abordagens) e um olhar refinado para as patologias especificas é uma boa combinação para iniciar uma intervenção efetiva..."

No que diz respeito aos locais de assistência, a atenção fornecida pelos terapeutas ocorre tanto em instituições de longa permanência, clinicas e hospitais, quanto em domicilio. Em casos de atendimento domiciliar é referida pelos entrevistados como atribuição do terapeuta ocupacional, verificar se existem condições mínimas de segurança para o idoso e, a partir da avaliação funcional do mesmo, identificar demandas comportamentais e relativas aos ambientes físico e social. Há que se ressaltar que em caso de proposição de adaptações ambientais e de dispositivos tecnológicos estes devem orientar-se para o respeito e favorecimento da autonomia. Segundo Diogo (2002), o ambiente não deve ser transformado, pois compõe a história do idoso. Acrescenta-se que o ambiente do idoso reveste- se de grande significado, compõe sua biografia podendo orientá-lo inclusive em momentos em que a memória falha, assim sugere-se somente proposição de mudanças que impliquem conforto e segurança ao paciente e favoreçam sua interação com o mesmo.

Em síntese, as abordagens utilizadas pelos entrevistados convergem para a redução da sintomatologia e preservação da independência e autonomia do idoso.

\section{CONCLUSÃO}

Pôde-se apreender, através do relato dos terapeutas, que as ações empregadas contribuem para amenizar a insegurança e o medo presentes nos idosos portadores de enfermidades crônicas degenerativas como a DP, minimizar sintomas e aumentar a independência e a autonomia. Os terapeutas ainda possuem em comum o respeito pelas pessoas que têm diante de si, considerando e valorizando características pessoais, história de vida e potencialidades das mesmas para superar dificuldades e buscar realizações; o propósito de prover seus clientes com atividades adequadas para o resgate e preservação da auto-estima e, o empenho na busca de meios, formas de tratamento e instrumentos diversos no sentido de aperfeiçoarem sua atuação profissional.

A partir do presente estudo identificam-se novos desafios tais como investigar em que medida as abordagens utilizadas colaboram para melhorar o estado geral do idoso e diminuir a assistência requerida.

ALMEIDA, M. H. M.; CRUZ, G. A. The interventions used by occupational therapist at the treatment of elderly with Parkinson's disease. Rev. Ter. Ocup. Univ. São Paulo, v. 20, n. 1, p. 29-35, jan./abr. 2009.

\begin{abstract}
The Occupational Therapy function and its objective with the elderly attacked by $\mathrm{PD}$, is to prevent and reduce functional losses and dependence frames. For it, this professional basically uses technological devices, environmental changes and facilitated techniques. A qualitative, investigative and descriptive research was developed in 2006 in order to know the procedures used by the Occupational Therapists with elderly attacked by PD. Through interviews with occupational therapists that work with gerontology; residents and active in the city of Sao Paulo, the research understood an application of a schedule of open questions. Seven occupational therapists have participated of the research. The interviewees relate that the elderly mainly face motor, cognitive and emotional difficulties and, guide themselves at their procedures according to the patients' desires and goals. These purposes take in minimize the symptoms, develop motor practice and skills, recover and increase remaining abilities, improve the performance in the daily activities, the independence and life quality. The developed procedures include motor coordination, techniques of relaxation and breath, compensated activities and the procedures development in order to the preserved capacities. To face the cognitive disturbs, the interviewee provide cognitive stimulation as far as simplification activities. To minimize the impact resulting from the disease increase is very important work with the careers and family. Summarize, the approach used by the interviewee focus to preserve the elderly independence, autonomy and significant life.
\end{abstract}

KEY WORDS: Aged. Occupational therapy. Parkinson disease. 


\section{REFERÊNCIAS}

ARAGON, A. Spotilight on occupational therapy. The Parkinson. PDS. Summer, 2008. Disponível em: <http://www.parkinsons.org. uk/PDF/Parkinson_taster_web.pdf>. Acesso em: 25 jun.2 009.

ALMEIDA, M. H.M. Validaçäo do instrumento CICAc: classificação de idosos quanto à capacidade para o autocuidado. São Paulo: s.n, 2003.

BARDIN, L. Análise de conteúdo. Lisboa: Edições 70, 1977.

BORSOI, S. A. T. Terapia ocupacional aplicada a gerontologia. In: PAPALEO NETTO, M. Gerontologia: a velhice e o envelhecimento em visão globalizada. São Paulo: Atheneu, 2000.

CABRAL, A. C. J.; STOPPE, A. J. Alterações psiquiátricas na doença de Parkinson. In: FORLENZA, O. V.; ALMEIDA, O. P. (Org.). Neuropsiquiatria geriátrica. São Paulo: Atheneu, 2001.

CHEN, L. A.; NEVES, S. R. Atenção à saúde do idoso com deficiência. COGest - Coordenação de Desenvolvimento da Gestão Descentralizada, 2002.

DIOGO, M. J. Formação de recursos humanos na área de saúde do idoso. Rev. Latinoam. Enferm, 2004.

GAUTHIER, L.; DALZIEL, S.; GAUTHIER, S. The benefits of group occupational therapy for patients with Parkinson's disease. Am. J. Occup. Ther., v. 41, n. 6, p. 360-365, 1987.

LANA, R. C.; GOULARD, F. R. P.; MAIA, T. A.; PRUDENTE, C; CARDOSO, F. Estudo da confiabilidade do questionário de qualidade de vida na doença de Parkinson - 39 (PDQ-39). In: ENCONTRO DE EXTENSÃO DA UFMG, 8., 2005. Anais. Belo Horizonte; 2005.

LITVOC J. Envelhecimento: prevenção e promoção da saúde. São Paulo: Editora Atheneu, 2004.

MINISTÉRIO DA SAÚDE. Fundação Oswaldo Cruz. Promoção da saúde. Brasília (DF), 1996.

MORA, R. M. La terapia ocupacional en el domicilio del paciente mayor. Disponível em: <http://www.terapia- ocupacional.com/>. Acesso em: 7 jun. 2006.

PARKINSON DISEASE SOCIETY PUBLICATION. Express yourself. The Parkinson. PDS, Summer, 2008.

PARKINSON'S DISEASE SOCIETY. How many people have Parkinson's [on-line]. London, 2009. Disponível em: <http://www. parkinsons.org.uk/about_parkinsons/what_is_parkinsons/how_many_ people_have_parkinson.aspx>. Acesso em: 13 mar. 2009.

PARKINSON DISEASE SOCIETY PUBLICATION. Towards a better future. PDS, November, 2005.

STOPPE, A. J. Característica clínica da depressão em idosos. In: FORLENZA, O. V.; ALMEIDA, O. P. de (Org.). Depressão e demência no idoso. São Paulo: Lemos Editorial, 1997. p. 47-68.

ZASLAVSKY, C.; GUS, I. Idoso: doença cardíaca e comorbidades. Arq. Bras. Cardiol., v. 79, n. 6, p. 635-639, 2002. 